\title{
Photonuclear reaction data and $\gamma$-ray sources for astrophysics
}

\author{
H. Utsunomiya ${ }^{1, a}$, S. Goko ${ }^{1}$, H. Toyokawa ${ }^{2}$, H. Ohgaki ${ }^{3}$, K. Soutome ${ }^{4}$, H. Yonehara ${ }^{4}$, S. Goriely ${ }^{5}$, P. Mohr ${ }^{6}$, and \\ Zs. Fülöp ${ }^{7}$ \\ 1 Department of Physics, Konan University, Japan \\ 2 Photonics Research Institute, AIST, Japan \\ 3 Institute of Advanced Study, Kyoto University, Japan \\ 4 JASRI/SPring-8, Japan \\ ${ }^{5}$ Institut d'Astronomie et d'Astrophysique, Université Libre de Bruxelles, Belgium \\ 6 Strahlentherapie, Diakoniekrankenhaus, Schwäbisch Hall, Germany \\ 7 ATOMKI, Debrecen, Hungary
}

Received: 31 July 2005 /

Published online: 24 February 2006 - C Società Italiana di Fisica / Springer-Verlag 2006

\begin{abstract}
Direct determination of photoneutron cross sections of astrophysical importance has recently become possible with use of quasi-monochromatic $\gamma$ beams produced in laser Compton backscattering (LCS) from relativistic electrons at AIST. The astrophysics to be discussed with the photodisintegration cross section are both stellar and big bang nucleosyntheses regarding the production of p-process and s-process nuclei as well as light elements. Synchrotron radiations from a 10 tesla superconducting wiggler (SCW) at SPring-8 serve as an ideal photon source to determine photoreaction rates. This paper covers the latest cross section measurements with the LCS photon beams and a feasibility study of determining $(\gamma, \mathrm{x})(\mathrm{x}=\mathrm{n}, \mathrm{p}, \alpha)$ reaction rates with the $\mathrm{SCW}$ radiation.
\end{abstract}

PACS. 25.20.-x Photonuclear reactions - 25.40.Lw Radiative capture - 26.30.+k Nucleosynthesis in novae, supernovae, and other explosive environments - 41.60.Ap Synchrotron radiation

\section{Introduction}

Recently real photon-induced reactions have attracted a revived interest in the context of nuclear astrophysics [1]. Currently efforts are made for determining photoneutron cross sections in the low-energy tail of the giant electricdipole resonance, laboratory reaction rates by photoactivation, and $E 1 \gamma$ strength function below the neutron threshold. Measurements of $(\gamma, \alpha)$ reactions may not be impossible though an intense photon source is required. At present all experimental efforts have, as a matter of course, been limited to stable nuclei; real photon-induced reactions on unstable nuclei are far beyond our scope.

The direct determination of photodisintegration cross sections utilizes quasi-monochromatic $\gamma$-ray beams from laser Compton backscattering (LCS) that have recently become available at AIST [2]. Photodisintegration measurements are characterized by simplification and accuracy by employing the LCS $\gamma$ beams, a bulk of target material, and a $4 \pi$-type neutron detector consisting of $\mathrm{BF}_{3} /{ }^{3} \mathrm{He}$ proportional counters embedded in a poly- ethylene moderator. It is interesting to note that electric giant dipole resonance (GDR) was systematically studied in

${ }^{a}$ e-mail: hiro@konan-u.ac.jp 1960s through 1980s with quasi-monochromatic photons from positron annihilation in flight in nuclear physics [3], whereas the GDR study is currently implemented by the LCS photons in nuclear astrophysics with emphasis on cross sections in the Gamow energy window for photonuclear reactions. The Gamow window lies immediately above the threshold energy in the neutron channel [4], while in the $\mathrm{p}$ or $\alpha$ channels it is shifted from particle threshold toward high energies for the Coulomb potential effect [5]. Some of the early measurements of GDR exhibit non-vanishing cross sections below neutron threshold, necessitating accurate measurements for nuclear astrophysics.

The astrophysics to be discussed with photodisintegration cross sections are both stellar and big bang nucleosyntheses: (a) p-process nuclei; (b) s-process nuclei; and (c) light nuclei. The group (a) is directly related to photonuclear reactions. In the group (b) for heavy nuclei with high level densities, photoneutron reactions are translated to neutron capture by the statistical model, whereas they are converted to radiative capture by the reciprocity theorem in the group (c) for light nuclei with low level densities.

In the p-process study, $(\gamma, \mathrm{n})$ cross sections provide strong constraints on the $E 1 \gamma$ strength function from 
which stellar reaction rates are calculated within the framework of the Hauser-Feshbach model [6]. Both photoproduction and photodestruction cross sections are needed to discuss the p-process origin; photodestruction is by far difficult to measure because of the low natural abundance of p-nuclei. The $E 1$ and $M 1 \gamma$ strength functions below neutron threshold in the tail of GDR are of unique astrophysical significance because they contribute to photodisintegration of nuclei in low-lying excited states that are thermally populated in stellar conditions. The stellar photodisintegration rate is larger by two to three orders of magnitude than the laboratory photodisintegration rate for a nucleus in the ground state. The reliability of the statistical model calculation is further enhanced if neutron optical potentials and nuclear level densities are constrained in different experiments like neutron capture, etc.

We limit the s-process study by means of photodisintegration to radioactive nuclei for which neutron capture leads to stable nuclei. In particular, photodisintegration is the most efficient way to constrain the neutron capture cross section for short-lived nuclei like ${ }^{185} \mathrm{~W}$ $\left(t_{1 / 2}=75 \mathrm{~d}\right)[7,8]$. There are many such short-lived nuclei to study. Recently, neutron capture cross sections are measured for ${ }^{151} \mathrm{Sm}$ with a half-life of 90 years at n-TOF of CERN [9]. It was proposed [10] to measure photodisintegration cross sections for ${ }^{152} \mathrm{Sm}$ by scanning across low-lying excited states of the residual ${ }^{151} \mathrm{Sm}$ nucleus in order to get insight into the stellar enhancement factor.

Photoneutron cross sections so far measured with the LCS $\gamma$ beams are categorized into the three groups as follows:

- (a) p-process nuclei: ${ }^{93} \mathrm{Nb},{ }^{139} \mathrm{La},{ }^{181} \mathrm{Ta}[6]$;

- (b) s-process nuclei: ${ }^{80} \mathrm{Se},{ }^{108} \mathrm{Pd},{ }^{141} \mathrm{Pr},{ }^{152} \mathrm{Sm}$, ${ }^{186} \mathrm{~W}[8,11],{ }^{187} \operatorname{Re}[11]$, and ${ }^{188}$ Os [11];

- (c) light nuclei: D [12], ${ }^{9} \mathrm{Be}[13]$.

A new photon source that is ideally suited to a systematic p-process study is under development at SPring-8 [14]. A beam of $8 \mathrm{GeV}$ electrons passing through a 10 tesla superconducting wiggler (SCW) produces high-energy synchrotron radiation that mimics blackbody spectra at temperatures of billions of kelvin. It is shown that this SCW radiation can be used to determine laboratory photoreaction rates not only for $(\gamma, \mathrm{n})$ but for $(\gamma, \alpha)$ and $(\gamma, \mathrm{p})$ reactions.

\section{Direct determination of $(\gamma, \mathbf{n})$ cross sections}

\section{1 p-process}

Among 35 nuclei classically referred to as p-nuclei, there are two rare odd-odd nuclei: ${ }^{138} \mathrm{La}(Z=57, N=81)$ and ${ }^{180} \mathrm{Ta}^{m}(Z=73, N=107)$. The p-process origin of ${ }^{180} \mathrm{Ta}$, the only naturally occurring isomer and the rarest nucleus in the solar system, was investigated with focus on its production cross-sections of ${ }^{181} \mathrm{Ta}(\gamma, \mathrm{n})^{180} \mathrm{Ta}[6]$. Using a stellar rate constrained by the experimental cross sections, a $25 \mathrm{M}_{\odot}$ type-II supernova (SN-II) model with solar metalicity has confirmed that ${ }^{180} \mathrm{Ta}^{m}$ is a natural product

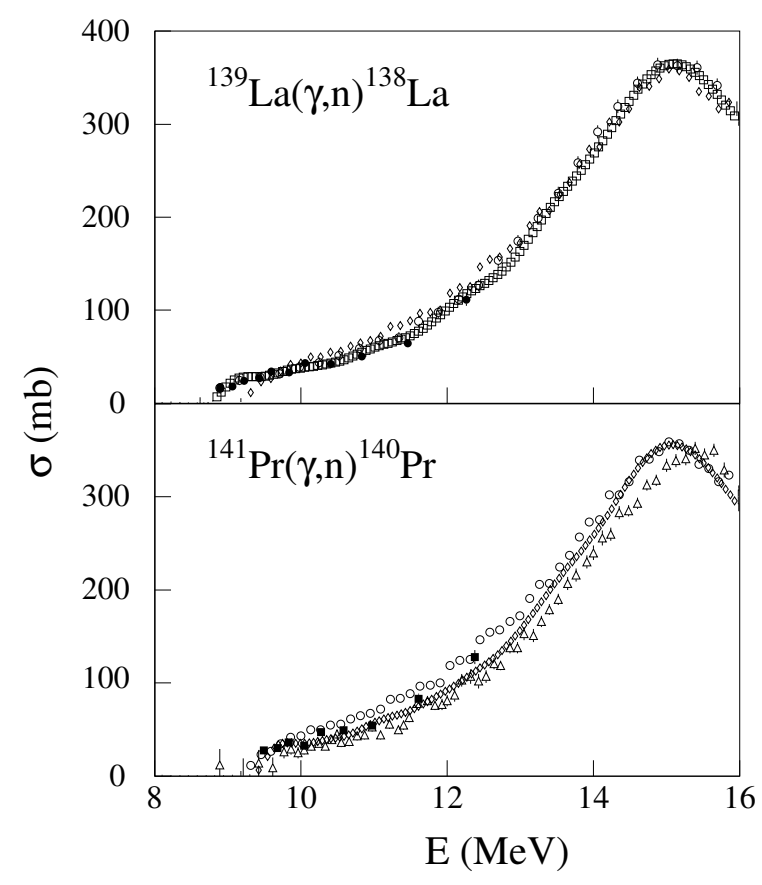

Fig. 1. Photodisintegration cross sections for ${ }^{139} \mathrm{La}$ and ${ }^{141} \mathrm{Pr}$ with the neutron magic number 82 (closed squares). Also shown are the previous data: open circles [19], open diamonds [20], and open squares [21] for ${ }^{139} \mathrm{La}$; open triangles [22], open circles [20], and open diamonds [21] for ${ }^{141} \mathrm{Pr}$.

of the p-process origin like the bulk of p-nuclides. Uncertainties remained both in experiment and theory: destruction cross sections of ${ }^{180} \mathrm{Ta}^{m}(\gamma, \mathrm{n})^{179} \mathrm{Ta}$ on one hand and contributions from $\nu_{e}$ captures on ${ }^{180} \mathrm{Hf}$ [15] as well as the controversial s-process origins $[16,17]$ on the other. The possibility of removing the experimental uncertainty is discussed in sect. 3.1.

Recently, cross sections for ${ }^{139} \mathrm{La}(\gamma, \mathrm{n}){ }^{138} \mathrm{La}$ were measured to address the underproduction problem of ${ }^{138} \mathrm{La}$ of thermonuclear origin in type-II supernovae (SNe-II) [18]. Figure 1 shows photoneutron cross sections measured for ${ }^{139} \mathrm{La}$ and ${ }^{141} \mathrm{Pr}$ with the magic neutron number 82 in comparison with the data taken previously [19,20,21,22]. The Hauser-Feshbach code MOST [23] with three different prescriptions of the $E 1 \gamma$ strength functions, namely the Lorentzian-type model [24], the Hybrid model [25] and the HFBCS + QRPA model [26] predicts a stellar rate $\lambda_{(\gamma \mathrm{n})}^{*}=27 \pm 15 \mathrm{~s}^{-1}$ for ${ }^{139} \mathrm{La}$ at a typical p-process temperature $T=2.5 \times 10^{9} \mathrm{~K}$. The upper limit of the rate $\left(\lambda_{(\gamma \mathrm{n})}^{*}=42 \mathrm{~s}^{-1}\right)$ is given by the Lorentzian-type model, while the lower limit $\left(\lambda_{(\gamma \mathrm{n})}^{*}=12 \mathrm{~s}^{-1}\right)$, which is the previous standard value [18], by the HFBCS + QRPA model. The large uncertainty arises from different energy dependences of the three different models of the $\gamma$ strength function below the neutron threshold.

To cure the underproduction problem completely within the p-process scenario of $\mathrm{SNe}-\mathrm{II}$, it is required that an increase in the production of ${ }^{138} \mathrm{La}$ needs to be 
complemented by a decrease in its destruction, resulting in a total enhancement factor of 20-25 [18]. The upper limit of the present rate may increase the stellar photoproduction rate of ${ }^{138} \mathrm{La}$ by a factor of 3.5 . The complementary decrease is however unlikely from the viewpoint of a consistency in calculating the production and the destruction with the same model; for example, the Lorentzian model of the $E 1 \gamma$ strength function, which could increase the production rate, increases the destruction rate simultaneously. Therefore, the p-process in $\mathrm{SNe}-$ II is not favored to explain a significant fraction of the solar ${ }^{138} \mathrm{La}$ abundance. However, no definite conclusion can be drawn until the destruction rate of ${ }^{138} \mathrm{La}$ is constrained experimentally. A future measurement of ${ }^{138} \mathrm{La}(\gamma, \mathrm{n})^{137} \mathrm{La}$ cross sections would be a challenge to experimentalists.

\section{2 s-process}

Photodisintegration cross sections were measured for ${ }^{186} \mathrm{~W},{ }^{187} \mathrm{Re}$ and ${ }^{188} \mathrm{Os}$, of which those for ${ }^{188}$ Os were used to address a major uncertainty involved in the ReOs cosmochronology [11]: namely, the correction factor $F_{\sigma}$ for the contribution of the $9.5 \mathrm{keV}$ state in ${ }^{187} \mathrm{Os}$ to the stellar neutron capture rate $[27,28,29,30]$. Figure 2 shows the present data in comparison with the data taken previously $[31,32,33]$. The result of the Hauser-Feshbach (HF) model calculations with different models of the $E 1$ $\gamma$ strength function, the level density, and the neutron optical potential are also shown in the figure. The HartreeFock + BCS (HFBCS) [34] and the back-shifted Fermi gas (BSFG) [35] models were used for the level density; The HFBCS + quasi-particle random phase approximation [26] (HFBCS + QRPA) and the Hybrid [25] models were used for the $\gamma$ strength function; The JLMB [36] and Woods-Saxon [37] optical potentials were used.

Stellar neutron capture cross sections for ${ }^{187}$ Os were calculated with the HF model parameters constrained by the present photodisintegration data and the laboratory neutron capture data available for ${ }^{187}$ Os. Note that in this case, the present data sensitive to the $E 1 \gamma$ strength function combined with the capture data sensitive to the neutron optical potential and the level density can greatly improve the reliability of the HF model calculations. Except for the one with the Woods-Saxon potential labeled INP-3 in fig. 2, which turned out to significantly underestimate the experimental capture cross sections at low energies, all the HF model calculations provided stellar capture cross sections with small deviations. Thus, it was concluded that $F_{\sigma}$ values at typical s-process temperatures $(12-30 \mathrm{keV})$ are in the range of $0.86-0.94$.

We investigated the uncertainty in the age of the Galaxy $\left(T_{G}\right)$ associated with the constrained $F_{\sigma}$ values in the Re-Os chronology. Using the simplest assumption of r-process nucleosynthesis yields of ${ }^{187}$ Re varying exponentially in time, the probable range of the differential coefficient $\mathrm{d} T_{G} / \mathrm{d} F_{\sigma}$ was found to be - (5.0-12.8) Gyr. Consequently, it was found that the associated uncertainty in $T_{G}$ values is less than $1 \mathrm{Gyr}$; when the temperature dependences of $F_{\sigma}$ and the Maxwellian-averaged cross sections

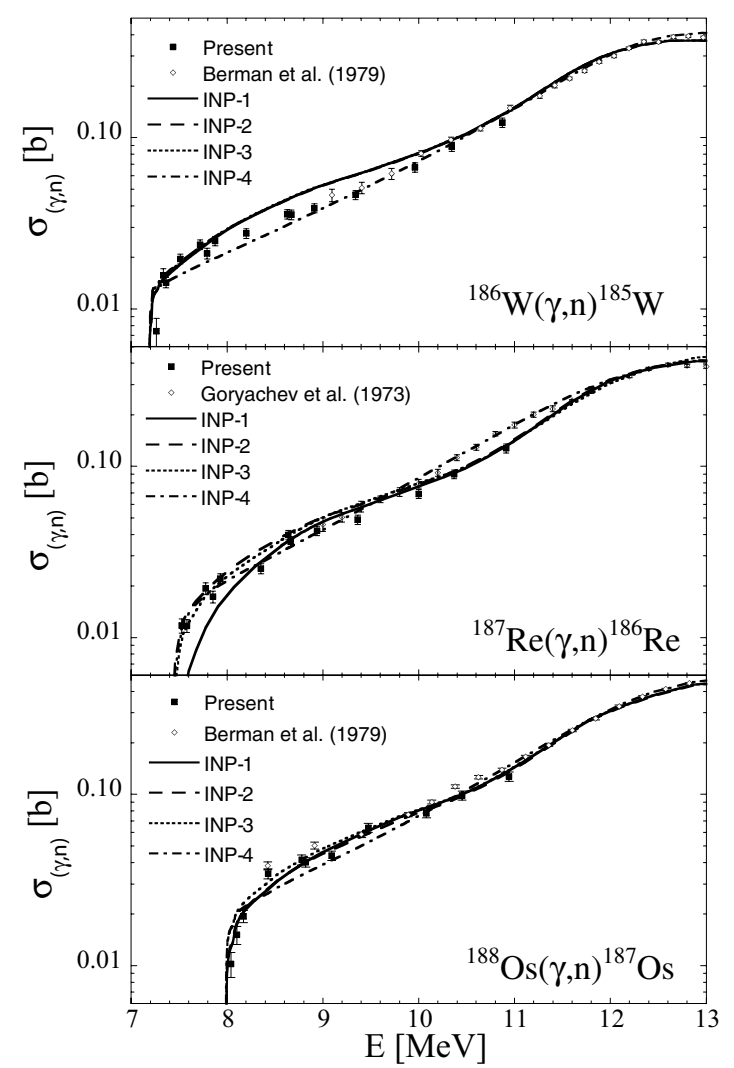

Fig. 2. Photodisintegration cross sections for ${ }^{186} \mathrm{~W},{ }^{187} \mathrm{Re}$, and ${ }^{188} \mathrm{Os}$ in comparison with the Hauser-Feshbach model calculations with different nuclear inputs. See text for details.

for ${ }^{186} \mathrm{Os}$ and ${ }^{187} \mathrm{Os}$ are considered, the uncertainties are less than 0.5 Gyr.

Nuclei at the s-process branching give a first approximation of the neutron density and temperatures of relevance in the s-process site [38]. Photodisintegration cross sections were measured for ${ }^{186} \mathrm{~W}$ to evaluate neutron capture cross sections for an s-process branching nucleus ${ }^{185} \mathrm{~W}$ $\left(t_{1 / 2}=75 \mathrm{~d}\right)$ [8]. The HF photodisintegration cross sections for ${ }^{186} \mathrm{~W}$ with two very different statistical ingredients [7] were scaled to the present experimental cross sections with scaling factors being 1.0 and 0.77 , respectively. By employing the same scaling factors for the HF neutron capture cross sections, a Maxwellian-averaged neutron capture cross section for ${ }^{185} \mathrm{~W}$ was evaluated; $\sigma=553 \pm 60 \mathrm{mb}$ at $k T=30 \mathrm{keV}$.

A discussion parallel to the previous classical-model analysis of the s-process flow at ${ }^{185} \mathrm{~W}$ gave a higher neutron density $N_{\mathrm{n}}=4.7_{-1.1}^{+1.4} \times 10^{8} \mathrm{~cm}^{-3}$. This neutron density, which is still compatible with the branching at NdPm-Sm ( $A=147-149)$ [39], highlights incompatibility with the branching at Er-Tm-Yb $(A=169-171)$ [39] and Os-Ir-Pt $(A=191-193)$ [40]. On the other hand, in a realistic s-process model the present neutron capture cross section for ${ }^{185} \mathrm{~W}$, which is smaller than the previous value $\approx 700 \mathrm{mb}$ [7], may enhance the overproduction of the sonly nucleus ${ }^{186} \mathrm{Os}$. 


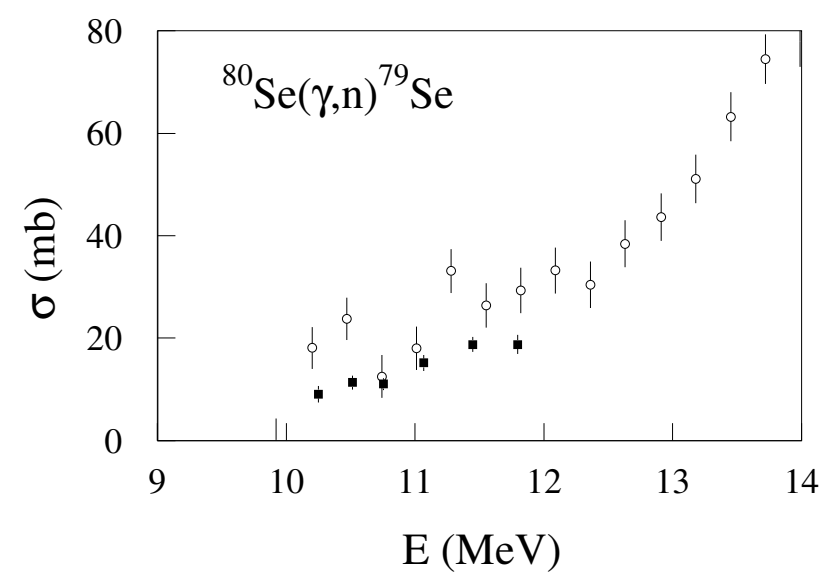

Fig. 3. Photodisintegration cross sections for ${ }^{80} \mathrm{Se}$ (closed squares). For comparison, the previous data (open circles) [42] taken with the positron annihilation $\gamma$-rays is also shown.

Long-lived radionuclides produced in the s-process are also of interest in the field of nuclear waste transmutation. The idea of nuclear transmutation is to transform them to either stable or short-lived nuclei by means of neutron capture. Photodisintegration can be used to evaluate neutron capture cross sections for nuclei in the former cases; they are ${ }^{36} \mathrm{Cl}\left[3 \times 10^{6} \mathrm{y}\right],{ }^{79} \mathrm{Se}\left[6.5 \times 10^{4} \mathrm{y}\right],{ }^{93} \mathrm{Zr}\left[1.53 \times 10^{6} \mathrm{y}\right]$, ${ }^{107} \mathrm{Pd}\left[6.5 \times 10^{6} \mathrm{y}\right],{ }^{151} \mathrm{Sm}[90 \mathrm{y}] .{ }^{151} \mathrm{Sm}$ and ${ }^{79} \mathrm{Se}$ are nuclei at which s-process branching takes place in the main and weak s-process flows, respectively. Note that the terrestrial decay rate of ${ }^{79} \mathrm{Se}$ is shortened by four orders of magnitudes in the stellar condition at temperatures higher than $1 \times 10^{8} \mathrm{~K}$ due to decay from excited states [41].

Photodisintegration cross sections were measured for ${ }^{80} \mathrm{Se},{ }^{108} \mathrm{Pd}$, and ${ }^{152} \mathrm{Sm}$ from the viewpoint of astrophysics and nuclear transmutation. Figure 3 shows results of the measurement for ${ }^{80} \mathrm{Se}$. The present data significantly differ from the data taken with the positron annihilation $\gamma$-rays [42].

\subsection{Light nuclei}

Photodisintegration of light nuclei is of particular interest because it is directly connected to inverse radiative capture by the reciprocity theorem. ${ }^{9} \mathrm{Be}$ and $\mathrm{D}$ are two good examples $[13,12]$; the inverse $\alpha \alpha(\mathrm{n}, \gamma){ }^{9} \mathrm{Be}$ reaction followed by ${ }^{9} \mathrm{Be}(\alpha, \mathrm{n})$ is considered to be most efficient in producing ${ }^{12} \mathrm{C}$ in the so-called neutrino-driven wind of SNe-II, dominating over the triple $\alpha$ reaction [43], while $\mathrm{p}(\mathrm{n}, \gamma) \mathrm{D}$ belongs to big bang nucleosynthesis, producing the simplest two-nucleon system. Photodisintegration is an exclusive way to study the three-body reaction, where real photons strongly excited the $E 1$ resonance state $\left(1 / 2^{+}\right)$in ${ }^{9} \mathrm{Be}$ immediately above the $\mathrm{n}+{ }^{8}$ Be threshold. In contrast, virtual photons in the electron backward-scattering favored M1 excitation [44]. On the other hand, it is a unique way to study the $\mathrm{p}(\mathrm{n}, \gamma) \mathrm{D}$ reaction, where the measurement successfully reduced uncertainties of $\mathrm{p}(\mathrm{n}, \gamma) \mathrm{D}$ cross sections at energies relevant to big bang nucleosynthesis.

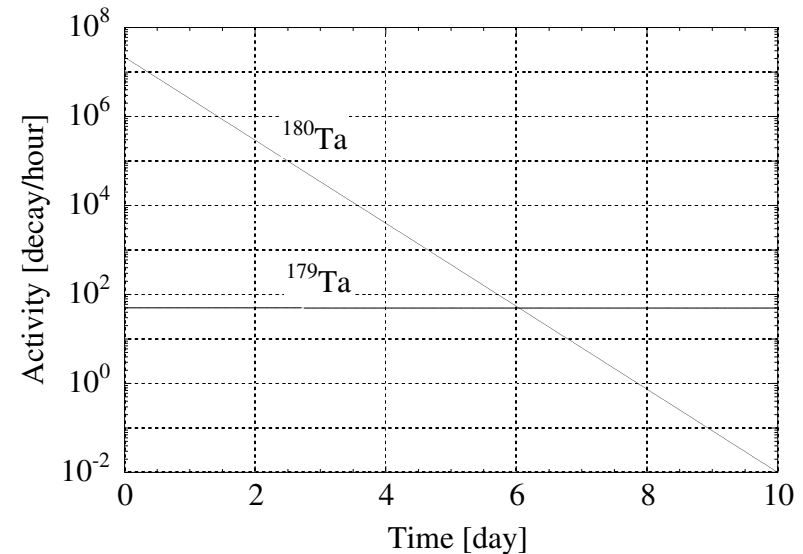

Fig. 4. The radioactivity of ${ }^{180} \mathrm{Ta}^{g s}$ and ${ }^{179} \mathrm{Ta}$ expected to be produced by irradiating 10 natural tantalum foils of $100 \mu \mathrm{m}$ thickness each with the SCW radiation for 100 hours.

New measurements are planned for photodisintegration of $\mathrm{D}$ to determine 1$)$ total $\mathrm{p}(\mathrm{n}, \gamma) \mathrm{D}$ cross sections at neutron energies below $100 \mathrm{keV}$ and 2) partial cross sections for s-wave and p-wave neutron capture. The former measurement is characterized by a long $\mathrm{D}_{2} \mathrm{O}$ target extending across the polyethylene moderator, intense LCS $\gamma$ beams with energies varied in a small step near the $n+p$ threshold, and a photon difference method for data reduction. Previously, a measurement of the photon analyzing power was carried out in photodisintegration of deuterons using linearly polarized $\gamma$ beams at the Duke facility [45]. The analyzing power data was used to determine relative $E 1$ and $M 1$ strengths of the photodisintegration cross section. In the new measurement, we plan to utilize linearly polarized LCS photons and fast (liquid scintillator) and slow $\left({ }^{3} \mathrm{He}+\right.$ polyethylene $)$ neutron detectors to determine absolute strengths of $M 1$ and $E 1$ cross sections from neutron angular distributions as well as the analyzing power.

\section{Perspectives}

\subsection{Determination of photodisintegration rates}

A 10 tesla superconducting wiggler is under development as an insertion light source at SPring-8. This light source produces high-energy synchrotron radiations with equivalent blackbody spectra at temperatures of billions of kelvin [14]. Recently, a study was made of using the SCW radiation to determine $(\gamma, \mathrm{n})$ reaction rates. Photodestruction of the nature's rarest isotope ${ }^{180} \mathrm{Ta}^{m}$ with the natural abundance of $0.012 \%$ will be the most challenging experiment. Irradiation of natural tantalum foils with the intense beam of the SCW blackbody radiation produces ${ }^{179} \mathrm{Ta}\left[t_{1 / 2}=1.82 \mathrm{y}\right]$ less by a factor of the order of 10,000 than ${ }^{180} \mathrm{Ta}^{g s}[8.15 \mathrm{~h}]$ because of the unbalanced natural abundances. Figure 4 shows the expected radioactivity of ${ }^{180} \mathrm{Ta}^{g s}$ and ${ }^{179} \mathrm{Ta}$ produced under a proper irradiation condition [14]. Because of the very different half-lives, the radioactivity of ${ }^{180} \mathrm{Ta}^{g s}$ decreases at the same level 
as ${ }^{179} \mathrm{Ta}$ in the first 6 days and is below $1 / 1,000$ in the next 4 days, while the activity of ${ }^{179}$ Ta remains the same ( $\sim 50$ decays per hour) during this period. After a proper cooling time of about 10 days, specific hafnium KX-rays $\left(\mathrm{K}_{\alpha 1}: 59.32 \mathrm{keV}, \mathrm{K}_{\alpha 2}: 57.98 \mathrm{keV}\right)$ emitted in the EC of ${ }^{179}$ Ta can be measured under a low-radiation background.

Very recently a feasibility study was extended to $(\gamma, \alpha)$ and $(\gamma, \mathrm{p})$ reactions. The event rate $N(t)$ is expressed by

$$
N(t)=a_{t} \int \sigma(\varepsilon) n_{\gamma}(\varepsilon, T) \mathrm{d} \varepsilon .
$$

Here $a_{t}$ is the areal number density of target nuclei $\left[\mathrm{cm}^{-2}\right]$, $\sigma(\varepsilon)$ is the photoreaction cross section for the emission of alpha particles or protons $\left[\mathrm{cm}^{2}\right], n_{\gamma}(\varepsilon, T)$ is a flux of the black-body radiation at temperature $T$ which is equivalent to the SCW synchrotron radiation $\left[\mathrm{s}^{-1}\right]$. Note that $T=4.4 \times 10^{9} \mathrm{~K}$ at the magnetic field of 10 tesla. The Gamow peak appears as a maximum in the integrand of eq. (1). The range of $\alpha$ particles and protons with the most probable energy defined at the Gamow peak was used to calculate $a_{t}$. Of the 233 reactions with cross sections compiled in [46] in the feasibility study, we found $35(\gamma, \alpha)$ and $36(\gamma, p)$ reactions with the event rate larger than 10 counts per hour. For example, in the ${ }^{96} \mathrm{Ru}(\gamma, \alpha){ }^{92} \mathrm{Mo}$ reaction the followings are obtained:

- The integral: $1.24 \times 10^{-21}\left[\mathrm{~cm}^{2} \mathrm{~s}^{-1}\right]$,

- The most probable $\alpha$ energy: $7.8 \mathrm{MeV}$,

- Range in Ru: $12.4[\mu \mathrm{m}]$,

- $a_{t}: 1.22 \times 10^{20}\left[\mathrm{~cm}^{-2}\right]$,

- Event rate: $5.43 \times 10^{2}\left[\mathrm{~h}^{-1}\right]$.

There are several interesting cases $\left({ }^{74} \mathrm{Se},{ }^{96} \mathrm{Ru},{ }^{144} \mathrm{Nd}\right.$, $\left.{ }^{152} \mathrm{Gd}\right)$, where a theoretical relation between $(\gamma, \alpha)$ and $(\alpha, \gamma)$ measurements can be investigated. Most of the $(\gamma, \alpha)$ reactions produce stable nuclei so that a direct counting of emitted $\alpha$-particles is necessary. The direct counting is possible by mounting target foils sufficiently thinner than the particle range inside a vacuum chamber and by surrounding each foil with particle detectors.

\subsection{Call for international collaborations}

Since photonuclear reactions have a variety of important facets in astrophysics, international collaborations in the following research categories can be called.

1) p-process: photodisintegration measurements, that are currently limited to $(\gamma, \mathrm{n})$ reactions, need to be extended to an unexplored field of $(\gamma, \alpha)$ and $(\gamma, \mathrm{p})$ reactions. Measurements of $E 1$ and $M 1 \gamma$ strength functions in nuclear fluorescence experiments should be addressed in the context of nuclear astrophysics: that is, photonuclear reactions on excited states under stellar conditions.

2) s-process: Photodisintegration plays a complementary role to neutron capture in the s-process study for radioactive nuclei. For example, a complementary role is seen in $(\gamma, \mathrm{n})$ for ${ }^{152} \mathrm{Sm}$ [stable] and $(\mathrm{n}, \gamma)$ for ${ }^{151} \mathrm{Sm}$ $\left[t_{1 / 2}=90 \mathrm{y}\right]$ as discussed in sect. 2.2. In particular, photodisintegration provides a unique opportunity to evaluate neutron-capture cross sections for short-lived nuclei provided that neutron capture leads to stable nuclei. One can find many nuclei in the chart of nuclides for a systematic study, e.g., ${ }^{64} \mathrm{Cu}[12.7 \mathrm{~h}]$, ${ }^{65} \mathrm{Zn}[244 \mathrm{~d}],{ }^{70} \mathrm{Ga}[21.2 \mathrm{~min}],{ }^{71} \mathrm{Ge}[11.4 \mathrm{~d}],{ }^{74} \mathrm{As}$ [17.8d] etc. Although the $E 1 \gamma$ strength function is best probed by photodisintegration, remaining sources of uncertainties in the statistical model calculation are the neutron optical potential and the nuclear level density. If these quantities could be probed in separate experiments, the predictive power of the statistical model would be greatly improved.

3) light nuclei: Photodisintegration of light nuclei is a straightforward way to study inverse radiative capture based on the reciprocity theorem. Deuterium is a good research objective, where $\sim 100 \%$ linearly-polarized photons can be used to separate the $E 1$ and $M 1$ components of photodisintegration and thus the corresponding s-wave and p-wave neutron captures by proton.

4) photon sources and experimental technique: There are four fundamental factors for photon sources: intensity, monochromaticity, polarization, and energy variableness. An attempt of producing monochromatic $\gamma$-rays at the Institute Laue-Langevin utilizes neutron-capture $\gamma$-rays produced at the reactor and a bent Si 220 crystal as a monochromator [47]. The 10 tesla superconducting wiggler at SPring-8 with another unique feature of being equivalent to blackbody radiation would allow one to determine photoreaction rates not only for $(\gamma, \mathrm{n})$ reactions [14] but also for $(\gamma, \alpha)$ and $(\gamma, \mathrm{p})$ reactions.

$5)$ theory and astrophysical modeling: Although important effort has been devoted in the last decades to measure reaction cross sections, theoretical predictions play a crucial role in the estimate of the reaction rates on stable as well as unstable nuclei for astrophysics applications. The nuclear ingredients to the reaction models, i.e. nuclear structure properties, optical model potentials, nuclear level densities, $\gamma$-ray strengths, should preferentially be estimated from microscopic global predictions based on sound and reliable nuclear models which, in turn, can compete with more phenomenological highly-parametrized models in the reproduction of experimental data. Well-targeted experiments are of prime importance to properly constrain theoretical models and consequently decrease the uncertainties in the predictions of astrophysics interest. A special care should be paid to the definition of the priorities and the sensitivity of the astrophysics observables to the nuclear ingredients. The relevance of the nuclear inputs should be dictated by astrophysics simulations which needs to be based on the state-of-theart models for each problem considered. A simultaneous effort to improve astrophysics models is required.

This work was supported by the Japan Society of the Promotion of Science (Grant-in-Aid for Scientific Research (B), (C)) 
and The Ministry of Education, Culture, Sports, Science, and Technology (Grant-in-Aid for Exploratory Research), and the Japan Private School Promotion Foundation. S.G. is FNRS Research Associate.

\section{References}

1. H. Utsunomiya, P. Mohr, A. Zilges, M. Rayet, to be published in Nucl. Phys. A, nucl-ex/0502011.

2. H. Ohgaki, S. Sugiyama, T. Yamazaki, T. Mikado, M. Chiwaki, K. Yamada, R. Suzuki, T. Noguchi, T. Tomimasu, IEEE Trans. Nucl. Sci. 38, 386 (1991).

3. S.S. Dietrich, B.L. Berman, At. Data Nucl. Data Tables 38, 199 (1988).

4. P. Mohr, K. Vogt, M. Babilon, J. Enders, T. Hartmann, C. Hutter, T. Rauscher, S. Volz, A. Zilges, Phys. Lett. B 488, 127 (2000).

5. P. Mohr, M. Babilon, D. Galaviz, K. Sonnabend, K. Vogt, A. Zilges, Nucl. Phys. A 719, 90c (2003).

6. H. Utsunomiya, H. Akimune, S. Goko, M. Ohta, H. Ueda, T. Yamagata, K. Yamasaki, H. Ohgaki, H. Toyokawa, Y.W. Lui, T. Hayakawa, T. Shizuma, E. Khan, S. Goriely, Phys. Rev. C 67, 015807 (2003).

7. K. Sonnabend, P. Mohr, K. Vogt, A. Zilges, A. Mengoni, T. Rauscher, H. Beer, F. Käppeler, R. Gallino, Astrophys. J. 583, 506 (2003).

8. P. Mohr, T. Shizuma, H. Ueda, S. Goko, Y. Makinaga, K.Y. Hara, H. Hayakawa, Y.-W. Lui, H. Ohgaki, H. Utsunomiya, Phys. Rev. 69, 032801 (2004).

9. U. Abbondanno et al., Phys. Rev. Lett. 93, 161103 (2004).

10. A. Mengoni, Proceedings of the International Conference on Nuclear Data for Science and Technology, Santa Fe, New Mexico (USA), 26 September - 1 October 2004, AIP Conf. Proc. 769, 1209 (2005).

11. T. Shizuma, H. Utsunomiya, P. Mohr, T. Hayakawa, S. Goko, A. Makinaga, H. Akimune, T. Yamagata, M. Ohta, H. Ohgaki, Y.-W. Lui, H. Toyokawa, A. Uritani, S. Goriely, Phys. Rev. C 72, 025808 (2005), nucl-ex/0506027.

12. K.Y. Hara, H. Utsunomiya, S. Goko, H. Akimune, T. Yamagata, M. Ohta, H. Toyokawa, K. Kudo, A. Uritani, Y. Shibata, Y.-W. Lui, H. Ohgaki, Phys. Rev. D 68, 072001 (2003).

13. H. Utsunomiya, Y. Yonezawa, H. Akimune, T. Yamagata, M. Ohta, M. Fujishiro, H. Toyokawa, H. Ohgaki, Phys. Rev. C 63, 018801 (2001).

14. H. Utsunomiya, S. Goko, K. Soutome, N. Kumagai, H. Yonehara, Nucl. Instrum. Methods A 538, 225 (2005).

15. S.E. Woosley, D.H. Hartmann, R.D. Hoffman, W.C. Haxton, Astrophys. J. 356, 272 (1990).

16. R. Gallino, C. Arlandini, M. Busso, Astrophys. J. 497, 388 (1998).

17. S. Goriely, N. Mowlavi, Astron. Astrophys. 362, 599 (2000).

18. S. Goriely, M. Arnould, I. Bolzov, M. Rayet, Astron. Astrophys. 375, L35 (2001).
19. R. Bergère, H. Beil, A. Veyssière, Nucl. Phys. A 121, 463 (1968).

20. H. Beil, R. Bergère, P. Carlos, A. Lepretre, A. Veyssière, Nucl. Phys. A 172, 426 (1971).

21. S.N. Beljaev, V.A. Semenov, Izv. Akad. Nauk SSSR 55, 953 (1991).

22. R.E. Sund, V.V. Verbinski, H. Weber, L.A. Kull, Phys. Rev. C 2, 1129 (1970).

23. S. Goriely, in Nuclei in the Cosmos, edited by N. Prantzos, S. Harissopulos (Editions Frontières, Gif-sur-Yvette, 1998) p. 314 .

24. C.M. McCullagh, M.L. Stelts, R.E. Chrien, Phys. Rev. C 23, 1394 (1981).

25. S. Goriely, Phys. Lett. B 436, 10 (1998).

26. S. Goriely, E. Khan, Nucl. Phys. A 706, 217 (2002).

27. S.E. Woosley, W.A. Fowler, Astrophys. J. 233, 411 (1979).

28. K. Yokoi, K. Takahashi, M. Arnould, Astron. Astrophys. 117, 65 (1983).

29. M. Arnould, K. Takahashi, K. Yokoi, Astron. Astrophys. 137, 51 (1984).

30. K. Takahashi, Nucl. Phys. A 718, 325c (2003).

31. B.L. Berman, M.A. Kelly, R.L. Bramblett, J.T. Caldwell, H.S. Davis, S.C. Fultz, Phys. Rev. 185, 1576 (1969).

32. A.M. Goryachev, G.N. Zalesnyi, S.F. Semenko, B.A. Tulupov, Yad. Fiz. 17, 463 (1973).

33. B.L. Berman, D.D. Faul, R.A. Alvarez, P. Meyer, D.L. Olson, Phys. Rev. C 19, 1205 (1979).

34. P. Demetriou, S. Goriely, Nucl. Phys. A 695, 95 (2001).

35. S. Goriely, J. Nucl. Sci. Tech. Suppl. 2, 536 (2002).

36. E. Bauge, J.P. Delaroche, M. Girod, Phys. Rev. C 63, 024607 (2001).

37. A.J. Koning, J.P. Delaroche, Nucl. Phys. A 713, 231 (2003).

38. F. Käppeler, H. Beer, K. Wisshak, Rep. Prog. Phys. 52, 945 (1989).

39. F. Käppeler, R. Gallino, M. Busso, G. Picchio, C.M. raiteri, Astrophys. J. 354, 630 (1990).

40. P.E. Koehler, J.A. Harvey, K.H. Guber, R.R. Winters, S. Raman, J. Nucl. Sci. Tech. Suppl. 2, 546 (2002).

41. K. Takahashi, K. Yokoi, At. Data Nucl. Data Tables, 36, 375 (1987).

42. P. Carlos, H. Beil, R. Bergère, J. Fagot, A. Lepretre, A. Veyssière, G.V. Solodukhov, Nucl. Phys. A 258, 365 (1976).

43. S.E. Woosley, R.D. Hoffman, Astrophys. J. 395, 202 (1992).

44. H.-G. Clerc, K.J. Wetzel, E. Spamer, Nucl. Phys. A 120, 441 (1968).

45. W. Tornow, N.G. Czakon, C.R. Howell, A. Hutcheson, J.H. Kelley, V.N. Litvinenko, S.F. Mikhailov, I.V. Pinayev, G.J. Weisel, H. Witala, Phys. Lett. B 574, 8 (2003).

46. T. Rauscher, F.-K. Thielemann, At. Data Nucl. Data Tables 88, 1 (2004).

47. P. Mutti, private communication. 